Classification

Physics Abstracts

$61.12-36.20-64.70 \mathrm{M}$

\title{
Chain conformation in nematic elastomers
}

\author{
H. Finkelmann ( $\left.{ }^{1}\right)$, W. Kaufhold $\left({ }^{1}\right)$, L. Noirez $\left({ }^{2}\right)$, A. ten Bosch $\left({ }^{3}\right)$ and P. Sixou $\left({ }^{3}\right)$ \\ (1) Institut für Makromolekulare Chemie. Universität Freiburg, 7800 Freiburg, Germany \\ ( $\left.{ }^{2}\right)$ Laboratoire Léon Brıllouin, CEN Saclay, 91191 Gif-sur-Yvette Cedex. France \\ (3) Laboratoire de Physique de la Matière Condensée, Parc Valrose, 06034 Nice Cedex, France
}

(Received 24 March 1993, revised 12 April 1994, accepted 4 May 1994)

\begin{abstract}
The anisotropy of the main chain conformation of a side-on fixed liquid crystal elastomer on application of a mechanical stress has been measured in the isotropic and nematic phases by small angle neutron scattering. The radii of gyration parallel and perpendicular to the stress field have been determined on elongation and as a function of temperature. A weak anisotropy of the main chain conformation is found only in the nematic phase at sufficiently high values of elongation. A comparison to a theory based on a Landau de Gennes expansion of the free energy for onented monodomain samples which includes coupling between elastic deformation and nematic strain is made.
\end{abstract}

\section{Introduction.}

Various liquid crystalline elastomers have been synthesized from crosslinked side chain liquid crystal polymers [1-14]. These materials exhibit exceptional physical properties due to the simultaneous presence of both rubber elasticity due to the crosslinked backbones and optical birefringence due to the mesogens on the side chains. Similar to an external electric or magnetic field, an elastic deformation of the network influences the alignment of the mesogens. A theory based on a Landau-de Gennes expansion of the free energy has been proposed for the isotropic phase [15] and has been used to explain the divergence of the stress optical coefficient as temperature is lowered towards the nematic phase as well as the shift of the transition temperature on elongation [1]. Structural studies on liquid crystal elastomers have also shown that a static stress field induces a molecular reorientation of the mesogenic units relative to the stress field. The alignment of the backbone chain appears to be low.

In this paper we present results on backbone anisotropy of a liquid crystal elastomer under mechanical stress. The method of small angle neutron scattering (SANS) was used for mixtures of hydrogenated elastomers with elastomers deuterated on the backbone. These experiments clearly show only a weak anisotropy of the conformation of the flexible chains on application of stress in the nematic phase. 


\section{Experimental.}

2.1 Synthesis, Phase behaviour, Crosslinking. - The synthesis of the liquid crystal (l.c.) elastomer is described in detail in reference [16] and outlined in figure $1(m=n=6)$. The monomer 1 (deuterated or hydrogenated) was prepared as described in the literature [1]. The linear copolymer 3 was synthesized by free-radical polymerization of the monomers 1 and 2 , where 2 is commercially available. The GPC molecular weight of the deuterated copolymer 3 before crosslinking is given as $M_{\mathrm{n}}=310000 \mathrm{~g} / \mathrm{mol}$ and $M_{\mathrm{w}}=610000 \mathrm{~g} / \mathrm{mol}$ (in $\mathrm{CHCl}_{3}$, polystyrene standard). The 1.c. elastomer 4 is obtained by crosslinking a $1: 1$ mixture of deuterated and hydrogenated methacrylate backbone of the linear copolymer 3 with 4,4methylenediphenyl isocyanate (MDI) as crosslinking agent. The phase behaviour of the linear copolymer 3 and 1.c. elastomer 4 was determined by polarizing microscopy and differential scanning calorimetry (DSC). Both the linear copolymer 3 and the 1.c. elastomer 4 show the same phase behaviour. The glass transition temperature $T_{\mathrm{G}}=30^{\circ} \mathrm{C}$ and the nematic-isotropic phase transformation temperature $T_{\mathrm{NI}}=60^{\circ} \mathrm{C}$ are not influenced by the weak crosslinking.

(CH

1<smiles>C=C(C)C(OCCO)OCCO</smiles>

2

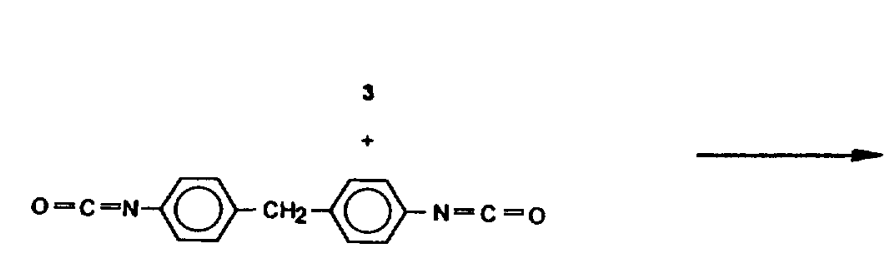

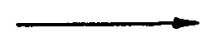

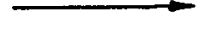$$
\text { (1) }
$$

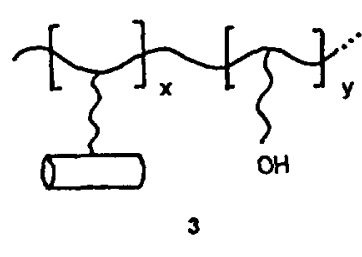

$x / y=0.96 / 0.04$

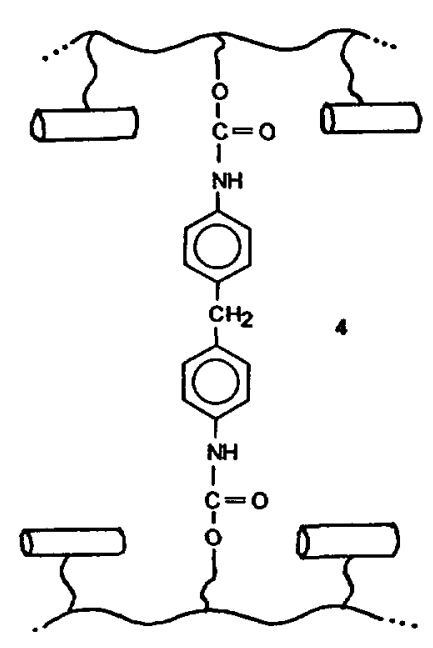

Fig. 1. - Chemical structure of the crosslinked nematic polymer. 
The crosslinking density of the elastomers was determined by swelling experiments in toluene and stress-strain measurements. In the swelling experiments, the average molar mass of the chains in the network, $M_{\mathrm{c}}$, was calculated from Flory's swelling theory, $M_{\mathrm{c}}=d_{\mathrm{e}} V_{1}(0.5-\chi)^{-1} \varepsilon^{5 / 3}$ with $d_{\mathrm{e}}$ the density of the elastomer, $V_{1}$ the molar volume of the swelling liquid, $q$ the equilibrium ratio of volumes of the swollen and unswollen samples and $\chi$ the Flory-Huggins parameter. The $\chi$ parameter was calculated from the second virial coefficient $A_{2}$ (obtained from light-scattering measurements [17]) using $\chi=0.5-A_{2} d_{\mathrm{p}}^{2} V_{1}$, where $d_{\mathrm{p}}$ is the density of the uncrosslinked polymer. Originally, $\chi$ describes exclusively the energetic interaction between solute and polymer. In reality $\chi$ includes an additional entropic component, which is not taken into account in the theory and $\chi$ should be treated as an adjustable parameter which depends on the specific properties of the particular polymersolvent combination [18]. Therefore, we should consider $M_{\mathrm{c}}$ not as an absolute value, but as a relative value dependent on the elastomer and the solvent.

The determination of $M_{\mathrm{c}}$ from mechanical measurements is based on the relation between stress and strain. For an ideal network, classical rubber elasticity theory predicts the relation : $\pi=\left(d_{\mathrm{e}} R T / M_{\mathrm{c}}\right)\left(\Lambda-1 / \Lambda^{2}\right)$ where $\pi$ is the nominal stress (force per unit unstrained crosssection) and $A=L / L_{0}$ the ratio of deformation. The value of $M_{\mathrm{c}}$ was obtained from the initial slope of the plot of $\pi v s$. $A-1 / A^{2}$ (see Ref. [1]).

The $M_{\mathrm{c}}$ values and the number of monomer units between two crosslinks, MU, from the deuterated and hydrogenated copolymer 3 are of the same order of magnitude (compare to Ref. [16]).

$\begin{array}{lcc} & M_{\mathrm{c}} & \mathrm{MU} \\ \text { From the swelling experiment : } & 180000 & 260 \pm 30\end{array}$

From the mechanical experiment : $137000 \quad 200 \pm 3$

\subsection{EXPERIMENTAL SET-UP.}

(1) Mechanical [16]. - The elastomer film was stretched symmetrically in the direction of the $z$-axis by two displacement disks, placed below and above the sample, working simultanously. The extent of elongation is given by $A=L / L_{0}, L$ being the length after stretching and $L_{0}$, the initial length. The sample was fixed at a given elongation in a closed heating chamber. The temperature was regulated to a constant value within $0.02 \mathrm{~K}$. The experiments were carried out statically after stabilization.

(2) Neutron scattering. - The SANS experiments were carried out at the Paxy spectrometer. The scattering intensities were obtained from the number of counts on an area detector. The range of accessible wave vectors is $5 \times 10^{-3}<q<3.5 \times 10^{-2} \mathrm{~A}^{-1}$. Here, the neutron beam is in the negative $y$-direction, the detector in the $x-z$ plane, the magnetic field in the $x$-direction, the elongation in the $z$-direction.

From the scattering image of the two-dimensional detector, the contributions $I_{z}(q)$ and $I_{\lambda}(q)$ (relative to the $z$ axis parallel to the elongation) were extracted and the plots of $I_{z}^{-1}(q)$ and $I_{\lambda}^{-1}(q)$ were traced as a function of $q^{2}$. The slopes determine the projections of the squares of the components $R_{z}$ and $R_{\lambda}$ of the radius of gyration obtained as a function of temperature and elongation (Tab. I). The zone for which $I_{1}^{-1}(q)=f n\left(q^{2}\right), i=x, z$, is linear is observed to extend beyond the limit of wave vectors of the order of the inverse of the apparent chain dimensions, possibly due to polydispersity [19]. As an example, in figure 2 we show inverse intensity plots versus squared wave vector $q^{2}$ in the isotropic $\left(70^{\circ} \mathrm{C}\right)$ and anisotropic $\left(47^{\circ} \mathrm{C}\right)$ phase for zero elongation. No anisotropy was found in either phase and no difference was observed between the $I_{r}^{-1}$ and $I_{r}^{-1}$ curves. 


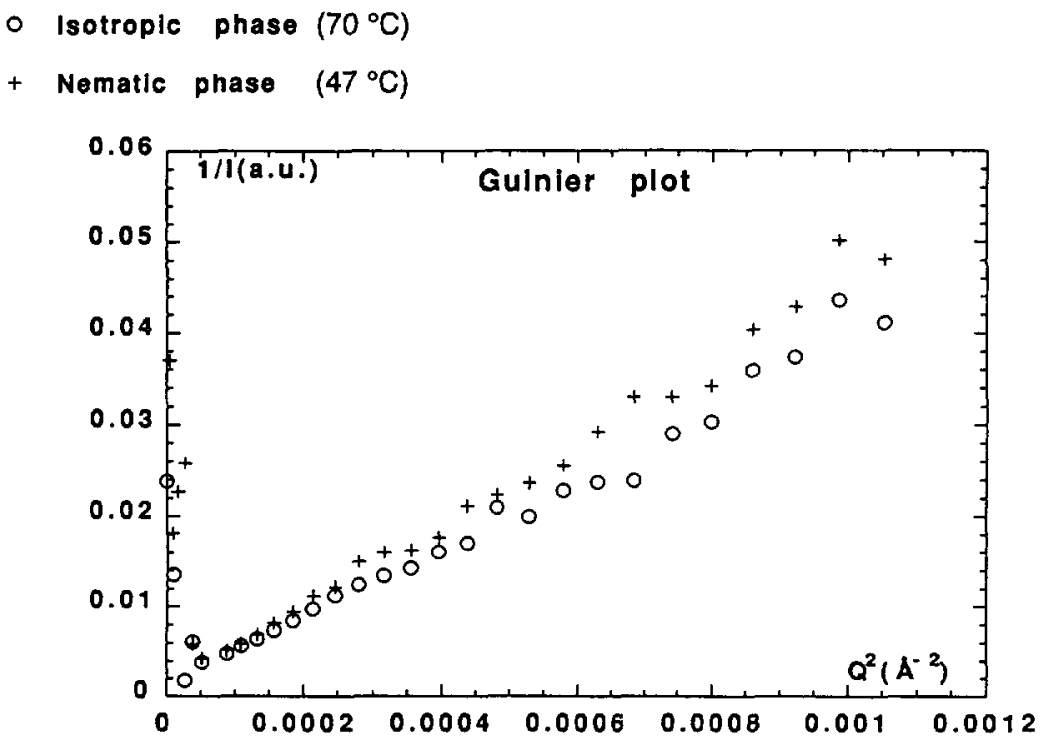

Fig. 2. - Plots of inverse scattering intensity, parallel or perpendicular to the direction of elongation, versus squared wave vector in the isotropic phase $(O)$ at $70^{\circ} \mathrm{C}$ and nematic phase $(+)$ at $47^{\circ} \mathrm{C}$ for zero elongation.

Relaxation experiments were also carried out (Fig. 3). The sample was elongated to $L / L_{0}=1.5$ and held at constant temperature. The mechanical stress was then removed and the chain dimensions were measured as a function of time as the sample relaxed to the unstrained state.

\section{Results.}

3.1 ZERO ELONGATION, EFFECT OF MAGNETIC FIELD. - In a magnetic field of $1.4 \mathrm{~T}$, no anisotropy was observed in the isotropic phase. In the anisotropic phase, the chain dimensions appear slightly elongated in the direction of the magnetic field. The effect of the magnetic field is weak. In the following results no magnetic field was applied.

The experimental results in zero magnetic field are given in table I and can be described as follows.

3.2 EFFECT OF ELONGATION : ISOTROPIC AND ANISOTROPIC PHASE. - In the isotropic phase, no anisotropy in the backbone chain dimensions on application of the uniaxial elongation is observed. The total radius of gyration $\left(2 R_{1}^{2}+R_{z}^{2}\right)^{1 / 2}$ increases. In the anisotropic phase, the chain conformation becomes anisotropic with $R_{z}>R_{1}$, or apparent stretching in the direction of macroscopic elongation with weak values of $R_{-} / R_{1}$ characteristic of uncrosslinked side chain polymers.

3.3 EFFECT OF TEMPERATURE AT CONSTANT ELONGATION. - The radius of gyration at zero elongation increases with decreasing temperature but no noticeable anisotropy occurs in the range of temperature considered.

For $L / L_{0}=1.5$, no anisotropy is found at high temperatures in the isotropic phase. On

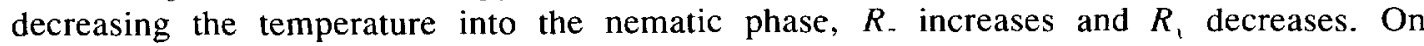
decreasing the temperature further, $R_{1} / R$. increases. 
- Riso $/ / 1.5$

O relax iso $/ 1$

Riso perp1.5

relax iso perp

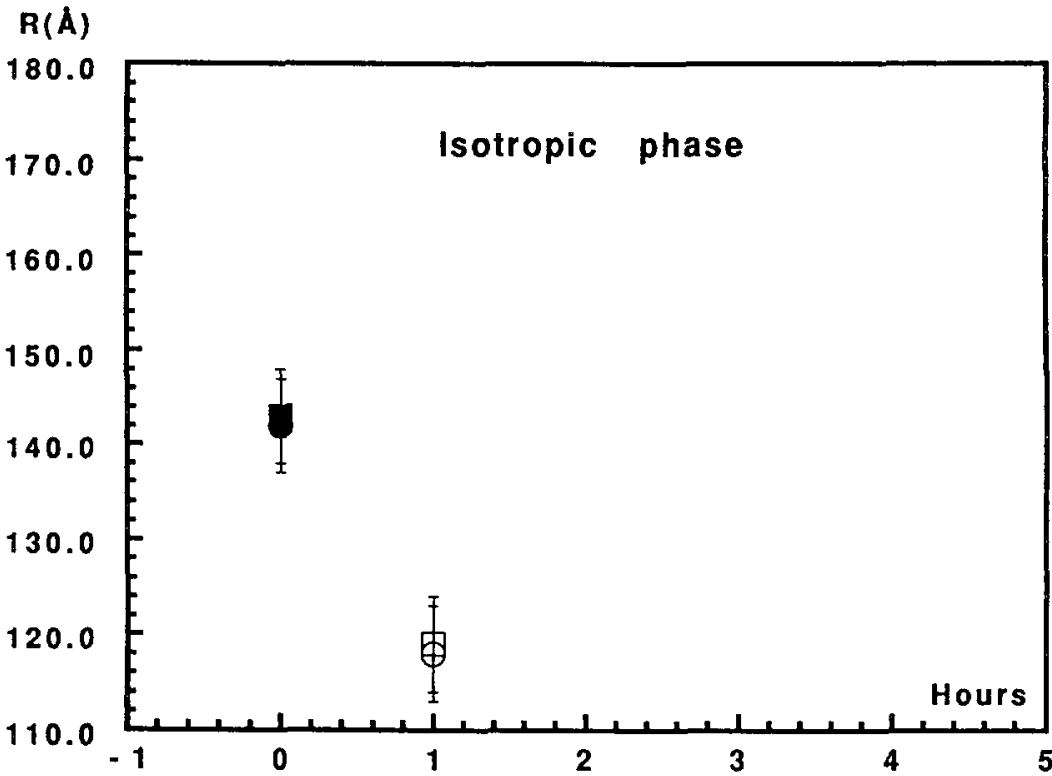

a)

N $1.547^{\circ}$ Cperp

relax $N 47^{\circ}$ Cperp

$\mathrm{N} 1.547^{\circ} \mathrm{C} / 1$

relax $N 47^{\circ} \mathrm{C} / /$

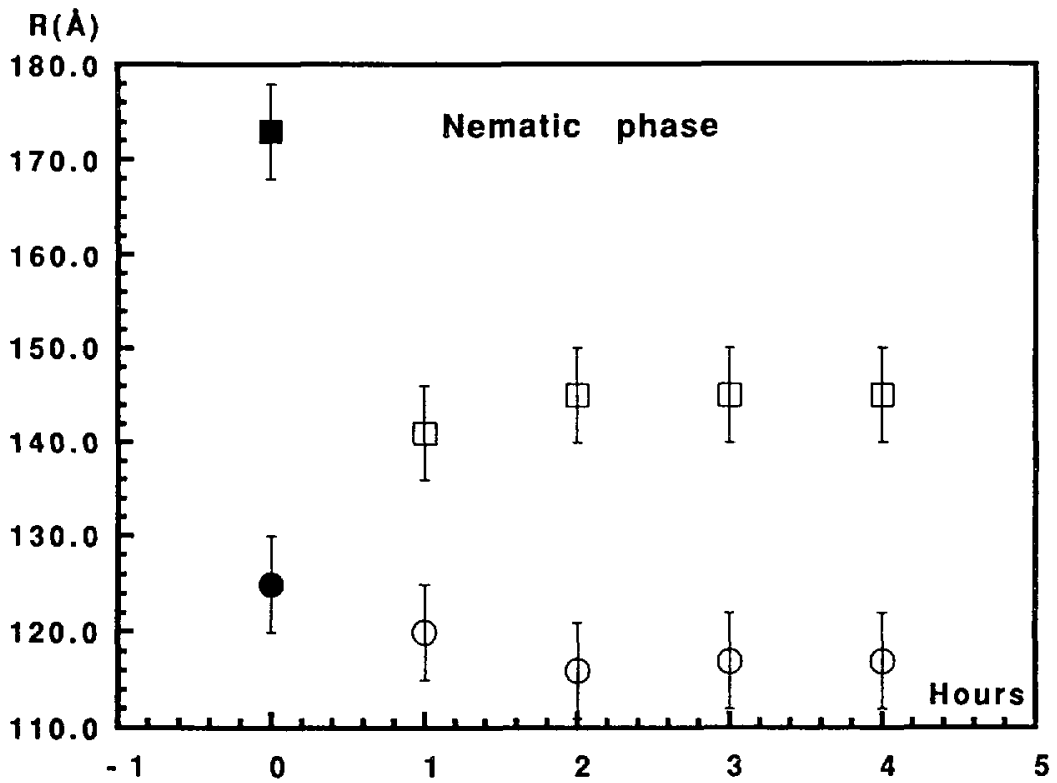

b)

Fig. 3. - Relaxation of the radius of gyratıon on removal of the uniaxıal elongation in the isotropic (a) and nematic phase (b) as a function of time. (4a) (घ) $R_{,}(\bullet) R_{1}$ : for $t=0$ with elongation $L / L_{0}=1.5 ;(\square) R_{z}(\mathrm{O}) R_{1}$ : relaxation. (4b)(ロ) $R_{\text {. }}(\bullet) R_{1}$ : for $t=0$ with elongation $L / L_{0}=1.5 ;$ (口) $R_{z}(O) R_{1}$ : relaxation. 
Table I. - Components of the radius of gyration of the polymer chain in a liquid crystal elastomer in the direction of elongation $\left(R_{z}\right)$ and in the perpendicular direction $\left(R_{1}\right)$ as a function of the elongation $L / L_{0}$ in the $z$-direction in the isotropic and nematic phases. The nematic-isotropic transition temperature is $60^{\circ} \mathrm{C}$.

\begin{tabular}{|l|l|l|l|l|l|}
\hline$T{ }^{\circ} \mathrm{C}$ & $L / L_{0}$ & Time & $R_{z}(\mathrm{~A})$ & $R_{1}(\mathrm{~A})$ & $R_{1} / R_{z}$ \\
\hline 70 & 1 & $5 \mathrm{~h}$ & 117 & 117 & 1 \\
47 & 1 & $5 \mathrm{~h}$ & 131 & 131 & 1 \\
70 & 1.5 & $5 \mathrm{~h}$ & 143 & 142 & 1 \\
46,6 & 1.5 & $5 \mathrm{~h}$ & 173 & 125 & 0.72 \\
32 & 1.5 & $5 \mathrm{~h}$ & 150 & 130 & 0.86 \\
\hline
\end{tabular}

3.4 Relaxation at CONSTANT temperature. - In the isotropic phase (Fig. 3a), a decay of the radius of gyration is observed as the main chains relax after about one hour to the unstressed state.

In the nematic phase (Fig. 3b), an initial decay saturates and the sample does not return to the initial values measured without strain within the time of observation (five hours). Slow equilibrium processes are typical of these materials and relaxation from the stress oriented monodomain sample to the initial field free polydomain structure appears to be not reversible. It is possible that the main chains relax fairly rapidly on removing the strain, and this short time behaviour is followed by a slow reorganization into polydomains of random orientation by motion of the mesogenic side chains.

\section{Theory.}

4.1 ElASTIC MODEL. - A model free energy density for these systems has been proposed [15] using a Landau-de Gennes expansion in the uniform nematic order parameter tensor $Q_{\imath \jmath}$, and includes the elastic energy due to the sample deformation tensor $e_{1}$ and coupling to the strain tensor

$$
\begin{aligned}
& F=F_{0}+A\left(T-T^{*}\right) Q_{\imath} Q_{\jmath}+B Q_{\imath} Q_{l k} Q_{k \ell}+C Q_{\imath \jmath} Q_{\jmath k} Q_{k \ell} Q_{\ell_{l}}+ \\
& +\mu e_{i j} e_{i j}+\frac{\lambda}{2} e_{11} e_{j j}-U Q_{11} e_{1 j}-\sigma_{1 j} e_{1 j} .
\end{aligned}
$$

$F_{0}$ is the energy of the undeformed isotropic phase, $\mu$ and $\lambda$ are the Lamé elastic constants, $U$ is a coupling constant, the external applied stress tensor is denoted by $\sigma_{\imath J}$. In addition to the original model, we include a bulk elastic coefficient and possible volume changes on stretching not usually considered in rubber elasticity $[18,20]$. The Poisson ratio is $\sigma=\lambda / 2(\lambda+\mu)$ and varies generally between 0 and 0.5 . Network functionality is not explicitly considered [18].

In the anisotropic phase, experiments $[1,16]$ and theory [21] show that the sample is initially in a disordered «polydomain » state and a transition to an ordered «monodomain » phase seems to occur on elongation. We can apply the model free energy (4.1) to the monodomain sample of uniform orientation.

In the nematic monodomain phase with the director parallel to the external stress, we derive the equations for the unknowns $e_{\lambda 1}, e_{y y}, Q_{x}, Q_{v y}, Q_{z z}$ and $\sigma_{z z}$ by minimization of equation (4.1) 
for a given elongation $e_{\cdots}$. The order parameter tensor is traceless, therefore

$$
Q_{11}+Q_{1 \imath}+Q_{z z}=0
$$

We investigate a solution symmetric with respect to rotation around the $z$-axis : $Q_{\lambda 1}=Q_{y v}, e_{\lambda \uparrow}=e_{y y}$.

We solve by linearization around the solution at zero mechanical stress : at $\sigma_{n n}=0$ the monodomain order parameter $Q_{z z}^{0}$ minimizes the free energy

$$
\frac{\partial F}{\partial Q_{z z}}=0
$$

In the monodomain sample, a spontaneous elastic deformation occurs due to the liquid crystalline interaction with

$$
e_{x}^{0}=-\frac{1}{2} e_{\cdots}^{0}
$$

and the elongation of the sample in the direction of the ordering of the liquid crystal units depends on the orientational order

$$
e_{--}^{0}=\frac{U}{2 \mu} \frac{(1-\sigma)}{(1-2 \sigma)} Q_{z:}^{0}
$$

For $\sigma_{z z} \neq 0$, we set $Q_{z z}=Q_{z z}^{0}+\delta Q_{z:}, e_{11}=e_{11}^{0}+\delta e_{11}$, and find that the stress induced order parameter is a linear increasing function of the stress induced elastic deformation with temperature dependence given by the free energy density $f$ obtained from equation (4.1) in the absence of elastic effects $f\left(Q_{\imath \jmath}\right)=A\left(T-T^{*}\right) Q_{\imath \jmath} Q_{\jmath}+B Q_{\imath \jmath} Q_{\jmath k} Q_{k \ell}+C Q_{\imath \jmath} Q_{\jmath k} Q_{k \ell} Q_{\ell_{\imath}}$. Then

$$
\delta Q_{z z}=\frac{U}{D}(1+\sigma) \delta e_{\ldots}
$$

with

$$
D=\frac{\partial^{2} f}{\partial Q_{z z}^{2}}\left(Q_{z z}^{0}\right)-U^{2} / 4 \mu .
$$

The sample deformation in the direction perpendicular to the force field is

$$
\delta e_{11}=-\left(\sigma+\frac{U^{2}(1+\sigma)}{4(\mu+\lambda) D}\right) \delta e_{-}
$$

This leads to the dependence of $\sigma_{z z}, Q_{z z}, e_{11}$ on $e_{-z}$ in monodomain liquid crystal elastomers given in figure 4 for various temperatures and for $\lambda=0$. Other values of the Poisson ratio between 0 and 0.5 were also considered but only small quantitative differences were found. The behaviour calculated by this model is quite different compared to isotropic elastomers and seems to be dominated not by elastic interactions but by induced liquid crystalline ordering (as, for example, in the second term of equation (4.8)).

In the monodomain phase, the elastic properties of the liquid crystal elastomer are determined by a Hooke law for the stress strain relationship. The apparent elastic constant depends not only on the isotropic elastic constant but also on the order parameter induced by the mechanical field and decreases with temperature. Extrapolation of the stress strain curves to zero stress determines the spontaneous deformation of the monodomain elastomer caused by anisotropic liquid crystal interactions as given by equations (4.4) and (4.5). 

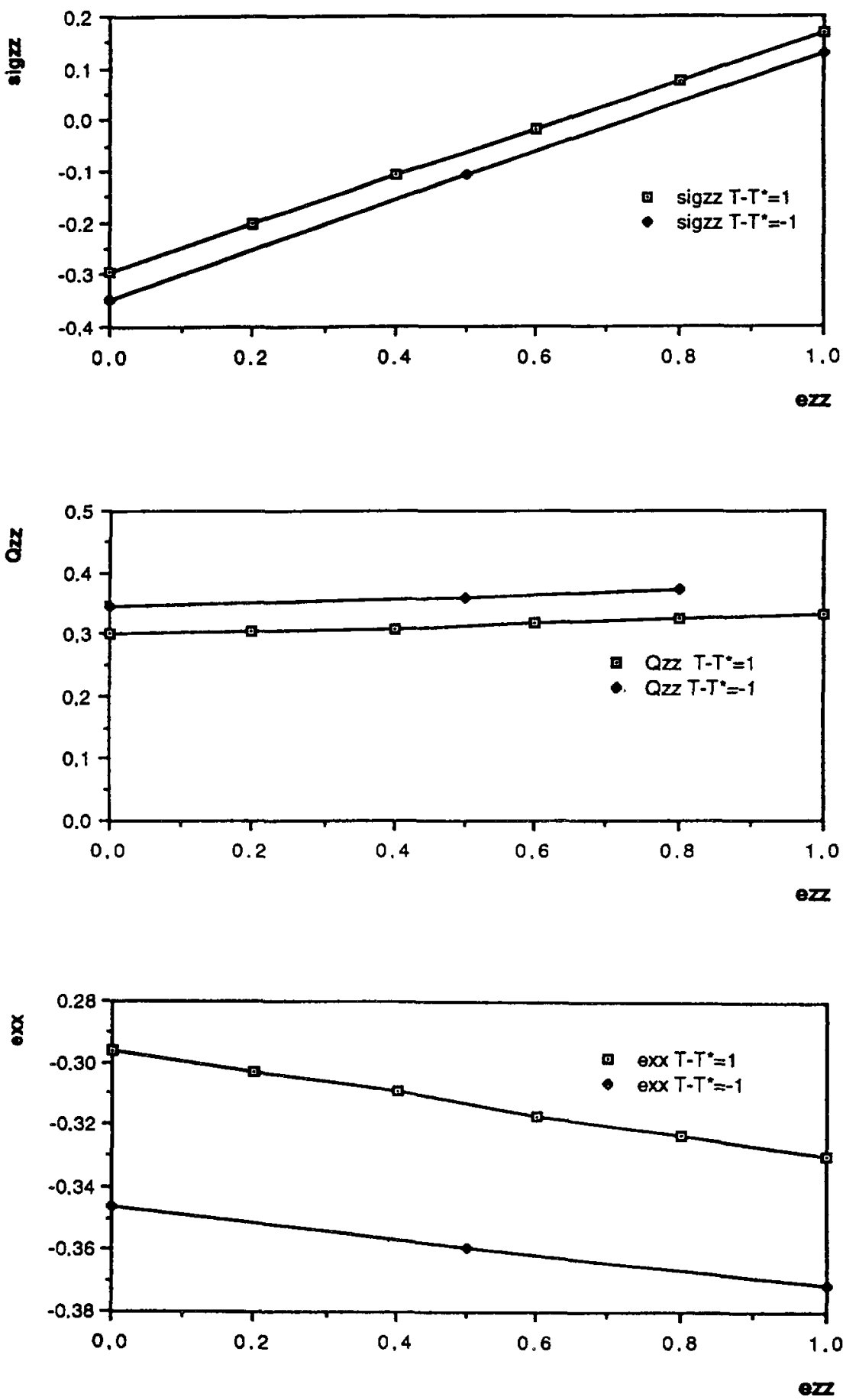

Fig. 4. - Calculated monodomain behaviour for the stress $\sigma .$. , the order parameter $Q_{z: \text {, and the }}$ perpendicular deformation $e_{11}$ as a function of the elongation $e_{z-}$ not considering the bulk elastic coefficient and for two different temperatures $T-T^{*}=1(\square)$ and $T-T^{*}=-1(\bullet)$. The values of the parameters of the free energy expansion are [16]: $A / U=0.8 \mathrm{~K}, B / U=-13.5, C / U=112.5$, $U / \mu=4$. 
The sample elongation, caused by the mechanical stress, induces a small order of the side chain mesogens in the monodomain sample, which increases with decreasing temperature.

The contraction of the sample perpendicular to the applied field seems to vary weakly on sample elongation and the sample dimensions in the $x$-direction decrease only slowly with increasing elongation in the $z$-direction and with decreasing temperature. This should lead to significant volume changes not usually found in elastomers.

\section{Discussion.}

The theoretical results presented in section 4 are in agreement [21] with experiments on the order parameter and the stress tensor as given in reference [1]. In the following, we will compare to the present work on the chain conformation in the isotropic and in the monodomain nematic phase.

In the initial polydomain phase at zero elongation, all directions of the director occur. This leads to nematic order and resulting chain extension locally in each domain but all such effects vanish globally after averaging over all the domain orientations [21]. The transition to a monodomain phase is expected to occur at a value of extension $L / L_{0}$ of about 1.2 [16]. Further ordering in the monodomain sample occurs then through orientation of the liquid crystal units relative to the uniform sample director.

In comparing theory to experiment, we identify [18] the microscopic deformation of the chain dimensions and the macroscopic sample deformation. This means that the components of length of each chain change in the same ratio as the corresponding bulk dimensions.

The spontaneous sample deformations at zero field, $e_{11}^{0}$ and $e_{z z}^{0}$, give an initial deformation of the bulk monodomain sample and an anisotropic conformation of the flexible chain in the monodomain nematic phase but vanish in the isotropic and in the initial polydomain sample. No anisotropy has been measured in the samples at zero external stress. The decrease of the average chain dimensions with increasing temperature could be due to increasing flexibility of the backbone chain (and smaller persistance length) in the bend elastic model [22], but experimental errors may surpass the $10 \%$ increase given in table I.

When the elastomer is deformed in the isotropic phase, ordering of the liquid crystal monomer units is induced and anisotropy of the backbone conformation is obtained from equation (4.7) and from (4.8) with, in this case, $D=18 A\left(T-T^{*}\right)-U^{2} / 4 \mu$ (for $T \gg T^{*}$ ). By use of the affine deformation assumption discussed previously [18], we identify

$$
\begin{aligned}
& \delta e_{--}=\left(L-L_{0}\right) / L_{0}=\left(R_{-}-R^{0}\right) / R^{0} \\
& \delta e_{11}=\left(R_{1}-R^{0}\right) / R^{0}
\end{aligned}
$$

The initial isotropic chain dimensions are given by $R^{0}$, the anisotropic components after deformation of the sample are $R_{1}$ and $R_{z}$. At $70^{\circ}$, an increase in chain dimensions but no appreciable anisotropy was measured at $L / L_{0}=1.5$ (Tab. I). Whereas from the theory, we expect elongation of the chain in the direction of the sample elongation and a weak contraction of the dimensions perpendicular to the external force.

When the sample is deformed in the nematic phase, a transition from polydomain to the monodomain structure has been calculated [21]. The total sample and chain deformation are then given by the spontaneous deformation (relative to the isotropic phase) due to the liquid crystal interaction and the deformation (relative to the monodomain sample at zero stress) due to the external field. From equation (4.5), the spontaneous deformations $e_{1,}^{0}$ and $e_{z:}^{0}$ are calculated to be similar in magnitude and to increase with decreasing temperature. These can be measured only in the stress free monodomain sample. The total deformation on application of a mechanical stress can be identified as before by using the chain dimension $R$ measured in 
the polydomain phase at zero stress, which eliminates the effect of anisotropic interactions. Then

$$
\begin{aligned}
& e_{-r}=\left(L-L_{0}\right) / L_{0}=\left(R_{-}-R\right) / R \\
& e_{\text {r }}=\left(R_{\text {๙ }}-R\right) / R .
\end{aligned}
$$

The agreement with experiment at $47^{\circ} \mathrm{C}$ and $L / L_{0}=1.5$ is within experimental error for the deformation of the chain in the $z$ direction and the deformation in the $x$ direction is found to be one order of magnitude smaller than $e_{z z}$. But as we expect $e_{11}$ to become more negative at lower temperatures, the ratio $R_{1} / R_{z}$ should decrease slightly (and the conformation should become more anisotropic) with decreasing temperature.

It would be of interest to relate the microscopic measurements on chain dimensions to measurements of the sample dimensions in both $z$ - and $x$-directions and this is currently in progress. A possible source of error could occur due to nonuniform deformation of the sample on elongation. The new results obtained in the present SANS studies require verification of the affine deformation assumed in these samples.

Memory effects have been demonstrated in liquid crystal elastomers particularily in the nematic phase and samples relax often very slowly or show irreversible behaviour. The path of experimentation can influence the value measured under given conditions as previous deformations are "frozen in " and cannot relax within the time of experimentation.

The samples used in the present studies were weakly crosslinked in the ordered phase but the crosslinking density could also be varied [16] and crosslinking could be performed either in the isotropic or in the ordered phase. The weak crosslinking and the low molecular weight of the present samples lead to weak values of the elastic constants and weak coupling of the chains to the elastic strain. The nematic field is active in causing anisotropic conformation of the flexible chains and the mechanical stress mainly acts as an aligning field for the transition to uniform or monodomain orientation.

In conclusion, the present preliminary results on the backbone chain conformation suggest that further experimentation is necessary to completely elucidate the mechanisms present in these novel elastomers. Notwithstanding, it appears that the behaviour of the backbone chain is more similar to that found in nematic side chain polymer melts in an orienting external field [22] than, as expected, in a rubber-like material.

\section{References}

[1] Schätzle J., Kaufhold W., Finkelmann H., Makromol. Chem. 190 (1989) 3269.

[2] Hessel F., Finkelmann H., Makromol. Chem. 189 (1988) 2275.

[3] Schätzle J., Finkelmann H., Mol. Cryst. Liq. Cryst. 142 (1987) 85.

[4] Zentel R., Benalia M., Makromol. Chem. 188 (1987) 665.

[5] Hessel F., Herr R. P., Finkelmann H., Makromol. Chem. 188 (1987) 1597.

[6] Hammerschmidt K., Finkelmann H., Makromol. Chem. 190 (1989) 1089.

[7] Gleim W., Finkelmann H., Makromol. Chem. 188 (1987) 1489.

[8] Talrose R., Gubina T., Shibaev V., Platé N., Makromol. Chem. Rap. Comm. 11 (1990) 67.

[9] Zentel R., Schmidt G., Meyer J., Benalia M., Liq. Cryst. 2 (1987) 651.

[10] Canessa G., Reck B., Reckert G., Zentel R., Makromol. Chem. Macromol. Symp. 4 (1986) 91.

[11] Davis F., Gilbert A., Mann J., Mitchell G.. J. Polym. Sci A 28 (1990) 1455.

[12] Schàtzle J., Ph. D. Thesis University of Freiburg, Germany (1989).

[13] Freidzon Y. S., Talrose R. V., Boiko N. I., Kostomin S. G., Shibaev V. P.. Platé N. A., Liq. Cryst. 3 (1988) 127. 
[14] Zentel R., Angew. Chem. Adv. Mater 101 (1989) 1437.

[15] de Gennes P. G., C.R. Acad. Sci. Paris 281 (1988) 101.

[16] Kaufhold W., Brand H. R., Finkelmann H., Makromol. Chem. 192 (1991) 2555.

[17] Richtering W., Schaetzle J., Adams J.. Burchard W., Colloid Polym. Sci., submitted for publication ;

Richtering W., Ph. D. Thesis Freiburg, Germany (1990).

[18] Treloar L. R., Physics of Rubber Elasticity (Clarendon Press, Oxford, 1975).

[19] Boué F., Adv. Polymer Sci. 82 (1987) 49; Ph. D. Thesis Université Paris-Sud (1982).

[20] Brand H. R., Rap. Comm. Makromol. Chem. 10 (1989) 57.

[21] Ten Bosch A., Varichon L., Die Makromolekulare Chemie, Theory and Simulation 3 (1994) 533.

[22] Varichon L., ten Bosch A., J Chem. Phys. 100 (1994) 1708. 